\title{
ENTRE SACRALISATION ET BANALISATION : LES ÉVOLUTIONS À LA TÉLÉVISION GRECQUE
}

\author{
Ioanna Vovou ${ }^{1}$
}

Les relations entre la politique et les médias consiste en une problématique aussi ancienne que les médias eux-mêmes. Les circonstances socio-historiques d'apparition des médias, ainsi que leur inscription dans la sphère publique en tant que structures institutionnalisées, offrent une clé d'entrée possible vers l'appréhension du phénomène. En ce sens, la télévision n'échappe pas à cette règle ; au contraire, elle semble nourrir davantage les questionnements et les approches scientifiques autour de la question. Dans le contexte d'un tel débat scientifique, mais aussi politique et social, qui s'instaure et prend de l'ampleur depuis une dizaine d'années environ, ce texte tente d'esquisser les caractéristiques de la médiatisation du politique à la télévision hellénique dans son évolution et d'analyser les tendances majeures de la représentation du discours politique télévisé qui s'avèrent en apparence, souvent, contradictoires.

\section{Mise en contexte historique de l'évolution du discours politique télévisé}

Le premier fil à suivre pour aborder et mettre en contexte l'évolution de la télévision grecque est intimement lié à la naissance

1 Ioanna Vovou est maître de conférences en sciences de l'information et de la communication à l'Université Paris 13.

Recherches en communication, $\mathrm{n}^{\circ} 24$ (2005). 
du média en grande partie sous un régime dictatorial. Les premiers pas de la télévision en Grèce se font dans une période tourmentée de la vie politique des années 60, juste avant le coup d'Etat des colonels. Quelle évolution pour un média dont les conditions de création s'inscrivent dans un contexte politique démocratiquement déficitaire ? La relation étroite entre pouvoir politique et télévision qui commence à se tisser dès cette époque, évolue, par la suite, dans un paysage audiovisuel déréglementé à partir du début des années 90 et connaît des mutations intéressantes jusqu'à aujourd'hui.

\section{Une expression politique télévisée en voie d'institutionnalisation}

Sous le régime des colonels du 21 avril 1967, la télévision a été considérée comme un moyen de propagande, de la même façon que la radio ${ }^{1}$ l'a été sous le régime de dictature de Ioannis Metaxas. (Papathanassopoulos, 1994 : 261). L'intervention gouvernementale sur la radiotélévision grecque et, en particulier, sur les programmes d'information a été constante pour la période qui a suivi la chute des colonels (après 1974), au point que cette dernière devienne un moyen de diffusion des idées et des décisions de chaque gouvernement. Les journaux télévisés ressemblaient plutôt à des porte-parole officieux et la censure était omniprésente et intense. De surcroît, la direction de la radiotélévision fut nommée (et est nommée jusqu'à aujourd'hui) selon ses affinités politiques et fut changée à chaque fois qu'un nouveau parti politique arrivait au pouvoir. Le contrôle imposé par chaque gouvernement était la raison pour laquelle, depuis l'époque de la restauration de la démocratie, les cadres supérieurs de la direction de la Radiotélévision grecque changent souvent.

Après la restauration de la démocratie en 1974, la télévision servira aux dirigeants politiques et, en particulier, au Premier ministre Constantinos Karamanlis, comme outil de diffusion de la position officielle de l'Etat. L'interview et le débat télévisés n'étaient pas encore établis comme formes légitimes d'information politique sur le petit écran. Cela est sans doute lié à la marge de liberté extrêmement restreinte dont le secteur audiovisuel bénéficiait. De cette façon, on a eu recours à des formes différentes d'intervention politique comme l'allo-

1 La naissance de la radiophonie publique a eu lieu en novembre 1935 avec la loi 19/20.11.1935 qui a créé le 'Service d'Emissions Radiophoniques'. Durant l'occupation allemande la radio était totalement contrôlée par les Allemands. 
cution, la transmission parlementaire, la "déclaration" et le "message"; puis la conférence de presse, davantage utilisé par Georges Rallis qui a succédé à C. Karamanlis. Par ailleurs, l'opposition n'eut guère accès aux ondes. (Psilla, 1990 : 770-780).

Les émissions existantes sur les ondes à cette époque avaient presque toujours la forme de débat entre plusieurs invités. Cela pourrait être interprété comme une sorte de prudence, le nombre d'invités fonctionnant en tant qu'amortisseur des affrontements directs sur l'écran. Comme le note Eric Darras, les rencontres télévisuelles des journalistes et des hommes politiques, ne doivent pas être considérées comme "naturelles"; tout au contraire, elles ont été historiquement constituées (Darras, 1997 : 11). Cette hypothèse est renforcée par le statut des invités choisis pour y participer. En effet, la plupart du temps, il s'agit des invités qui jouent, certes, un rôle dans le processus politique ou civique, mais qui ne font pas partie des acteurs de premier plan, comme ce serait, par exemple, le cas des ministres. De plus, l'idée d'interviewer le Premier ministre ou le Président de la République dans le cadre d'une émission de ce type n'était même pas envisageable, car le dialogue était exclu des pratiques de communication politique des années 70 . En outre, les thèmes des débats portaient sur des sujets politiques au sens large du terme, comprenant des thèmes sociaux, économiques et civiques.

En ce qui concerne le discours politique médiatisé, nous pouvons dire qu'avec l'avènement du PASOK au gouvernement en 1981, la télévision cesse d'être un simple accessoire de la scène politique et devient partie intégrante du jeu politique. (Psilla, 1990 : 782-789). La télévision est de plus en plus sollicitée pour exprimer les positions du gouvernement à travers de formes diverses : l'interview, la conférence de presse, le "discours en public", la transmission parlementaire, la "déclaration politique", etc. De même, le genre du débat politique télévisé fait ses premiers pas timides durant cette période. Les émissions politiques commencent à avoir une place de plus en plus régulière dans la programmation des chaînes publiques (notamment sur ET1) et ont une durée de vie plus longue. Le Premier ministre Andreas Papandreou était la figure dominante de la scène télévisuelle, comme le fut auparavant et à sa manière Constantinos Karamanlis. Progressivement, les dialogues et les débats entre des journalistes et des hommes politiques directement liés au pouvoir exécutif deviennent la règle d'une émission politique. La présence sur les plateaux des ministres du gouvernement du PASOK et des représentants des autres partis politiques qui acceptaient 
d'être interrogés par des journalistes, représenta un nouveau mode de communication et de médiation politique.

\section{Déréglementation du paysage télévisuel dans les années 90}

Durant les années 1980, le paysage audiovisuel a connu un changement spectaculaire dans sa structure dû à une nouvelle idéologie politique qui encourageait l'entrée du secteur privé et limitait le pouvoir de l'Etat. De cette façon, la radiotélévision a été considérée comme une nouvelle source d'investissement et de profit. Le changement des systèmes audiovisuels en Europe a été appelé « déréglementation ». En Grèce, il est aussi appelé « libération » ${ }^{1}$, ce qui est révélateur des conditions politico-idéologiques de l'époque. La création des deux premières chaînes privées, Mega et Antenna², est considérée comme emblématique, marquant le passage à une nouvelle période télévisuelle grecque. Les interviews politiques diffusées par les médias audiovisuels qui sont apparues avec la création de la radiophonie privée, ont pris une ampleur considérable durant les premières années de création des chaînes privées de télévision. Par ailleurs, il a été noté qu'en termes quantitatifs, l'offre de l'information sur l'ensemble du programme des chaînes augmente, en comparaison avec les années précédentes, puisque la durée d'émissions télévisuelles s'étend considérablement. (Pleios, 2002 : 268-269).

La création et l'établissement d'autres chaînes privées ont permis aux émissions d'interview et de débat de connaître une évolution remarquable. Dorénavant, toutes les chaînes -y compris celles du service public- vont inclure une, deux, voire plusieurs émissions politiques dans leur programmation hebdomadaire. Malgré l'existence occasionnelle des interviews politiques durant le monopole de l'ERT (Radiotélévision Publique Grecque), nous pouvons considérer que l'interview politique télévisée en tant que genre journalistique légitime est relativement récente en Grèce. Dans une ambiance où télévision privée rimait avec liberté, l'interview politique face à face a été privilégiée pendant les premières années qui ont suivi la déréglementation des ondes hertziennes. Pour la première fois, les hommes politiques étaient

1 Terme utilisé dans la langue courante et dans plusieurs ouvrages sur le sujet, notamment dans le livre de Papathanassopoulos Stylianos (2004) qui a comme titre : En libérant la télévision.

2 Mega commence officiellement sa diffusion le 20 novembre 1989 et Antenna le 31 décembre 1989, le soir du réveillon. 
à la disposition des journalistes, en face d'eux, pour répondre à leurs questions. Le "face à face" présenta la garantie d'un affrontement direct à travers lequel la vérité surgirait. Après le règne des émissions d'interview politique, les émissions de débat prennent en grande partie le relais, mais dans une optique légèrement différente de celle rencontrée dans les années 70 qui consistait à amortir les affrontements. Le débat à plusieurs, dirigé par un meneur de jeu incarné dans l'image du présentateur, a été considéré comme un modèle dialectique plus propice pour la production ou l'accouchement de la vérité. Les nombreuses possibilités de mise en scène et de mise en spectacle (direct, duplex, reportages, intervenants spéciaux...) sont devenues impératives pour répondre à la demande d'audience et à la concurrence ; par conséquent, elles n'allaient pas rester inexplorées.

Dans une tentative de comparaison avec les premiers traitements du discours politique dans les débats des années 70, nous observons aussi certains changements formels. Par exemple, l'interview "face à face" avec un homme politique est considérée comme trop dangereuse et n'est pas envisagée, en général, à la télévision jusqu'à la fin des années 80. Après cette date, les "face à face" avec des hommes politiques font leur apparition à la télévision grecque et auront une évolution considérable. De nos jours, le recours au "face à face" avec une femme ou un homme politique répond plutôt à des besoins d'atteindre une audience maximale, profitant de la notoriété de l'invité (voir plus loin). Par ailleurs, la présence sur les plateaux des émissions dites politiques des personnalités célèbres n'est pas un phénomène nouveau, puisqu'il existe aussi avant l'avènement de la télévision privée pour des raisons différentes, à savoir l'impossibilité d'interroger les dirigeants politiques. Dans le courant des années 90, ce choix de diversification des thèmes et des invités en dehors du champ politique stricto sensu, renvoyant partiellement à des glissements normatifs du genre, est soustendu par la stratégie de programmation et d'offre de programmes par les chaînes, privilégiant la dilution de l'objectif politique dans celui du débat social, voire "mondain" ou "people". En dernier lieu, le processus de morcellement du marché audiovisuel grec qui s'est accentué à partir de la deuxième moitié des années 90 et la multiplication des chaînes privées remodèlent le paysage audiovisuel, indiquant des stratégies différentes de la part des entreprises médiatiques en matière d'offre de programmes. 


\section{Tendances actuelles de la mise en scène du politique}

Nous avons observé jusqu'à ce stade de notre bilan de l'évolution de la médiation télévisuelle du discours politique que l'apparition des personnalités politiques à la télévision dans des formes autres que l'allocution débute après la chute de la dictature en 1974. Il faudra, grosso modo, attendre la fin des années 80, et plus encore les années 90 avec l'avénement de la télévision privée, pour que l'interview politique et le débat, prennent entièrement leur place dans la programmation des chaînes et pour qu'on puisse débattre avec le personnel politique de premier plan. Dans cet ordre d'idées, l'introduction de la communication et du marketing politique, en même temps que le morcellement du marché télévisuel grec, se fait progressivement ressentir, notamment après les élections de 2000 et, plus encore, après celles de 2004.

Dans la tentative de dresser un constat ponctuel de la situation actuelle en matière de représentation du discours et des personnalités politiques à la télévision grecque, nous mettrons l'accent sur un certain nombre de dispositifs télévisuels utilisés au service de la mise en scène du politique.

\section{De la «glorification » des personnalités politiques...}

\section{L'interview-profil}

Une des tendances qui perdure le plus dans la tradition de sollicitation des représentants politiques, surtout de premier plan, consiste en la valorisation de leur personnage par l'intermédiaire des interviews "face à face". On dresse le profil de l'invité politique à travers son parcours, ses positions et opinions. Dans ce cas, le ton du discours peut prendre des allures d'émissions "people" (qui dressent souvent le profil des invités), sous des apparences "sérieuses" et respectables. Ceci fait partie d'une culture de forte "personnalisation" des enjeux et du jeu politique, qui perdure dans la tradition politique grecque.

À titre d'exemple, l'émission À travers les yeux d'Elli, diffusée sur la chaîne privée Antenna de 1999 jusqu'à aujourd'hui, est assez illustrative. En premier lieu, déjà, le titre renvoie à l'idée de vérité, que le téléspectateur arrive à voir à travers les yeux de la journaliste tel un témoin oculaire ${ }^{1}$ de la vérité, du moins symboliquement. En deuxième

\footnotetext{
1 Cf. Jost, $2001:$ 62-78.
} 
lieu, l'affichage d'une approche "directe" refusant la langue de bois est une intention qui se manifeste même verbalement par la journaliste Elli Staï qui présente l'émission : «...Ceci signifie que vous les [femmes et hommes] politiques devez vous décider à parler une autre langue, n'est-ce pas ? » (émission du 5 janvier 2000). L'approche directe, voire spontanée, de la réalité est accentuée par un style d'énonciation, des questions et, en général, une parole qui se veulent pragmatiques sans être trop familiers. Le ton de la confidence est, également, de rigueur. Par exemple, dans l'émission citée ci-dessus, s'adressant à Dora Bakoyanni, députée du parti de droite Nea Dimocratia, devenue maire d'Athènes en 2002, la journaliste affirme : «je peux vous dire quelque chose, Nea Dimocratia semble un parti fatigué... ».

Ce "parler vrai" s'étend aux domaines extérieurs à la sphère publique et politique, revendiquant le droit à une vérité concernant l'ethos du personnage qui se construit pleinement en ayant recours à son univers personnel. Au fil du déroulement de l'interview, nous basculons d'une émission d'actualité politique vers une discussion de "femme à femme". Dans l'effort de présenter un portrait "complet" de la femme politique que la journaliste a devant elle, après les sujets politiques, on a droit à toute une série de questions sur la vie privée de la députée. Tour à tour l'on voit traiter l'invitée dans sa dimension de femme. Par exemple : «[...] je voudrais vous demander combien votre vie personnelle après votre mariage a changé votre façon d'agir en politique ? Êtes-vous plus relaxée, voyez-vous d'un autre point de vue les choses ? » Après la réponse affirmative de Dora Bakoyanni acceptant naturellement la question et insistant sur le besoin d'équilibre psychique ${ }^{1}$, des questions concernant son statut de mère complètent le portrait. Puis, s'enchaînent des questions portant sur sa famille, d'une part sur ses parents (Dora Bakoyanni étant la fille de l'ancien Premier ministre Constantinos Mitsotakis, figure importante de la scène politique grecque depuis les années 50) et d'autre part, concernant les valeurs et l'ambiance familiale chez les Mitsotakis. Ainsi, le portrait valorisant d'une femme comblée nous est dessiné. Dora Bakoyanni est présentée comme une femme dynamique, heureuse dans sa vie personnelle, mère et membre d'une famille équilibrée ayant des traditions solides de bonne entente et de dialogue derrière elle, caractéristiques

1 La députée rappelle qu'elle avait perdu cet équilibre dans sa vie personnelle pendant plusieurs années, en faisant allusion à l'assassinat de son mari Pavlos Bakoyannis par l'organisation terroriste "17 novembre". 
dont on imagine les vertus une fois étendues à l'ensemble de la société. La députée elle-même accepte volontiers de se prêter à ce jeu de mise à nu de sa vie intime et familiale, tout en utilisant l'argument par son genre $^{1}$ quand elle explique sa vision du fonctionnement de son parti politique : « ...Car tous ensemble nous pouvons offrir quelque chose, alors que chacun tout seul ne peut pas, Madame Staï ; c'est la simple vérité. Vous pouvez me dire que c'est une logique féminine, mais c'est la vérité ».

Les glissements entre la sphère publique et la sphère privée, ainsi que la redéfinition des frontières et de la relation entre les deux notions (Pleios, 2001 : 410), deviennent de plus en plus fréquents dans la représentation médiatique du processus politique, souvent sous la forme de ce dispositif d'interview "face à face", très utilisé pour interroger des personnalités connues dans tous les domaines publics. Nous notons toutefois le fait que cette tendance prend surtout forme dans les paroles de femmes journalistes et politiques. Le fait, d'une part, que ce type de questions personnelles ne soit pas posé, à notre connaissance et jusqu'à présent, à des hommes politiques (on parle rarement d'un homme "comblé" ou "complet") et, d'autre part, qu'une femme journaliste ne tienne pas tout à fait le même discours face à un invité homme nous conduit, en outre, à entrevoir une expression médiatique sexuée (Vovou, 2004).

\section{La construction de symboles}

Un autre dispositif audiovisuel utilisé dans la représentation des personnalités politiques et leur action est le reportage. Mis à part sa vocation informative, une fois inclus dans les émissions d'actualité ou de débat politique, l'usage du reportage sert, à son tour, à la création d'une ambiance, soumise souvent à des logiques affectives, plutôt qu'à des développements argumentatifs. L'utilisation de ces récits télévisés consiste essentiellement et dans certains cas à ajouter une dimension émotive.

Le reportage peut servir de socle à la célébration d'un certain nombre d'acteurs de la société et de la politique hellénique. Ce chemin qui mène à la focalisation in extremis sur "les" acteurs principaux est, certes, parfois dicté par l'actualité ou l'invité choisi, mais traduit néanmoins un choix et une façon réductrice d'appréhender le monde et

1 Au sujet de l'argumentation par le genre explicite et implicite, voir Bonnafous, 2002 et 2003 . 
l'Histoire en tant qu'actes individuels. En ce sens, les émissions consacrées au décès des personnalités importantes, par exemple, mettent en place tout un cérémonial, dans lequel le reportage n'est qu'une partie d'un dispositif submergé par le pouvoir émotif du direct. De tels exemples sont nombreux dans différents programmes (émissions politiques, magazines d'information, journaux télévisés). Il s'agit du cas où le passé semble "marquer" le présent et l'avenir avec une sorte d'encre indélébile. Par exemple, le traitement de la maladie, de l'hospitalisation et de la mort d'Andreas Papandreou en 1995, Premier ministre à l'époque, est assez emblématique de ce moment critique de l'évolution historique et politique du pays, où le respect et le recueillement était imposé par tous les journaux télévisés de $20 \mathrm{~h} 30$ pendant une longue période.

Toutefois, la glorification via les images est aussi réservée aux hommes politiques en bonne santé, avec un présent et un avenir politique. Tel est le cas, par exemple, de l'émission La pomme de discorde consacrée au Congrès général du PASOK, après la mort d'A. Papandreou, diffusée le 3 juillet 96. L'invité de l'émission est le ministre des travaux publics, de l'aménagement du territoire et de l'environnement de l'époque, Costas Laliotis. Au début de l'émission, un extrait de son discours lors du Congrès, consistant en l'appel au rassemblement des membres du parti, est repris. Cet extrait rappelle l'ambiance surchargée du Congrès, au sein duquel le message unificateur prononcé par $\mathrm{C}$. Laliotis donne à cet homme politique une image positive ${ }^{1}$. Sur le même ton, le numéro de L'heure de vérité du 20 mai 98 clôt l'émission sur une vidéo rassemblant les moments heureux du mandat du Maire d'Athènes, Dimitris Avramopoulos, qui était l'invité de l'émission. Etant donné que l'émission a eu lieu quelques mois avant les élections municipales, ce document très flatteur contribue à l'image et à la campagne du maire d'Athènes.

1 Dans le cadre de ce Congrès du PASOK, au gouvernement à l'époque, qui s'est déroulé en juin 96, la question de la présidence du parti après la mort d'A. Papandreou avait soulevé de fortes tensions. Le Premier ministre Costas Simitis avait déclaré qu'il démissionnerait de son poste s'il n'obtenait pas la présidence du parti, tandis qu'Akis Tsohatzopoulos plaidait pour une sorte de double autorité, séparant la présidence du parti des fonctions du Premier ministre et se présentant lui-même comme candidat à la présidence du PASOK. Les membres du parti étaient divisés en deux camps, donnant, encore une fois, la preuve du rôle de la polarisation en tant que schéma stratégique des affrontements politiques, même à l'intérieur des partis politiques. La fin du Congrès a donné la présidence au Premier ministre C. Simitis. 
De cette façon, s'opère un glissement du sujet à l'invité, entrâ̂nant une personnification et, souvent, une simplification des enjeux au "tempérament" des dirigeants politiques, favorables à la célébration implicite de ces derniers.

\section{... À leur « détrônisation » apparente}

\section{La mise en spectacle sur l'écran}

Parallèlement à la tendance de "glorification" du personnel politique de premier plan, l'on pourrait observer une apparente désacralisation et une certaine banalisation du discours politique. En effet, les personnalités politiques sont invitées fréquemment (presque quotidiennement) à participer à des émissions et à des journaux télévisés, face à des journalistes ou à des adversaires politiques sous l'enseigne de la polémique. Le dispositif scopique qui a la valeur d'une règle généralisée dans les émissions de plateau à la télévision grecque est celui du duplex et du multiplex, divisant l'écran télévisuel en plusieurs compartiments. Ce dispositif considérablement spectaculaire, appelé en Grèce "les fenêtres"1, insiste sur l' aspect fortement virtuel du studio de certaines émissions et journaux télévisés. Ces derniers ne représentent plus aux yeux du téléspectateur un topos réel, tangible où des personnes se réunissent pour discuter. Il correspond à un espace virtuel permettant la connexion simultanée avec d'autres espaces, et en cette qualité il se rapproche davantage d'un réseau de paroles, plus qu'à un lieu. Dans cet ordre d'idées, J.-P. Esquenazi évoque la notion de réseau et s'appuie, essentiellement, quoique pas exclusivement, sur la théorie de Michel Serres, inspirée de l'abstraction mathématique. Le réseau est ainsi défini comme un diagramme contenant des sommets individualisés, possédant leur "puissance propre" et des chemins transportant "des flux de détermination différents". Parmi les caractéristiques des réseaux nous retrouvons un parcours tabulaire et non dialectique, et la multiplication de types de relations qui ne sont, pourtant, pas liées entre elles suivant une logique de causalité. Par ailleurs, le réseau imposerait une logique

1 La journaliste Elli Staï a "importé" en Grèce ce dispositif de duplex et de multiplex lors de sa collaboration avec la chaîne télévisée SKAÏ en 1993-94, professant que la «croyance dans le pouvoir du direct » constitue «l'âme» du débat télévisé, une règle à laquelle toutes les composantes se plient. (Entretien accordé à l'auteur, in Vovou, $2000: 283$ ). 
du regard qui se traduit dans l'équation voir $=$ savoir $^{1}$. Il s'agirait plus d'une opération de lecture que de vision, ce qui conduit Serres à établir que le réseau utilise un langage purement informationnel, la dimension symbolique étant gommée. De cette façon, la complexité des problèmes de sens est réduite à la forme d'un réseau. Dans ce contexte, le réseau, par sa mobilité, «n'existe que sur le mode de l'actualité » et «n'a aucune mémoire » (Esquenazi, 1999 : 191-196).

\section{La dilution du politique et l'élargissement des sujets}

Par ailleurs, ce qui s'observe, en particulier ces dernières années, est la dilution du caractère purement politique des émissions de débat qui s'affichaient comme telles au départ. Ceci s'opère pour des raisons diverses, notamment d'audimat, mais aussi à cause d'un rapport quelque peu différent à la politique, influencé, selon certains chercheurs, par le contexte d'une société de consommation au sein de laquelle l'information politique ne serait pas reçue littéralement mais en se référant à un univers symbolique donné (Pleios, 2001 : 451). En ce sens, l'apparition des hommes politiques sert à valoriser et légitimer l'émission en rappelant sa vocation initiale. Dans les mêmes émissions, on voit apparaitre des comédiens et des artistes de tous ordres. Toutefois et de façon générale, on ne mélange pas le personnel politique avec les invités d'autres domaines dans le même numéro et dans ce type d'émissions de débat.

La tendance qui consiste à élargir les sujets et les invités au-delà du contexte politique stricto sensu ne fait, donc, aucun doute, en tant que pratique suivie par les émissions de débat. Même si ce phénomène semble généralisé et est perçu comme une tendance nouvelle, il est pourtant présent, du moins dès le début des années 1990, quand les émissions de débats ont acquis une stabilité dans la programmation des chaînes. D'innombrables exemples d'interviews effectuées par des journalistes politiques avec des personnalités du show biz, des chanteuses et des chanteurs desdites « grandes pistes » du pays (salles de spectacles musicaux), des acteurs, pour en citer les plus sollicités, témoignent de la solidité d'un dispositif, initialement conçu pour les fonctions précises du débat politique. Pourtant, les normes de conversation interpersonnelle ayant un fond commun et malgré l'éventail large de leurs applications, le dispositif de débat politique permet l'éloignement des

1 Ici Esquenazi (1999 : 196) se réfère à Musso P., Télécommunication et philosophie des réseaux, PUF, 1997, p. 99. 
thèmes habituels, sans la mise en cause des émissions. Les objections éventuelles concernant l'atteinte à leur légitimité sont repoussées par les présentateurs, soucieux de justifier leurs choix ${ }^{1}$.

Les sujets non politiques sont, également, contrebalancés par le poids médiatique des invités et leur légitimité dans leur propre champ, qu'il s'agisse d'art, de musique, de sport, etc. Par cette attitude, il est sous-entendu que toute opinion est digne d'être écoutée, du moment où elle est proférée par n'importe quelle «excellence civile» (cf. Esquenazi, 1999 : 333). Mais ce qui est plus important pour le maintien de la légitimité propre de l'émission est sa concordance avec l'actualité à chaque fois qu'il y a besoin. En effet, aucune émission politique ne privilégie une thématique autre que politique lors d'une actualité correspondante ${ }^{2}$. De cette façon, une émission n'est pas qualifiée de politique quand la totalité des sujets traités ne concerne pas le champ politique. En fait, la définition du genre est plus large. La pratique montre que les émissions politiques grecques obtiennent leur légitimité selon leur capacité de répondre à l'actualité avec des invités politiques, chaque fois qu'il est nécessaire. Dans la même tendance d'une dilution des sujets politiques dans l'agenda de l'actualité établi par les journalistes, l'on retrouve la participation des personnalités politiques dans les émissions matinales d'information. Opérant comme des magazines d'information plus ou moins généralistes, ces programmes invitent sur le plateau des invités en provenance de la sphère publique, dont des représentants politiques ${ }^{3}$.

1 De nombreux présentateurs de télévision attribuent cette tendance à l'incapacité de l'actualité politique grecque de fournir des thématiques sur une base hebdomadaire et au nombre limité des personnages politiques capables de tenir un discours intéressant à la télévision (propos recueillis des journalistes N. Hatzinikolaou, E. Staï et déclarations de la journaliste O.Tremi lors d'un séminaire à l'Université Panteion en 1995). D'un autre coté, Grégory Derville (1997: 113-114) parle du « temps de l'actualité sur lequel se calent les médias, hebdomadaire ou quotidien, (qui) correspond mal au temps dans lequel s'inscrit l'action politique, beaucoup plus tournée vers le moyen ou le long terme ». L'auteur soulève le problème de l'existence de deux temporalités différentes, l'une correspondant à une logique de programmation fidélisante et à une sérialisation, propre à une attitude consommatrice et l'autre, correspondant au travail dans les lieux du politique.

2 Il est vrai, par exemple, que les débats avec des gens du milieu artistique sont plus abondants pendant les fêtes de Noël et celles du premier de l'an, les émissions tentant de s'aligner au calendrier, à la temporalité et à la disponibilité de réception du public.

3 De telles émissions d'information matinales se retrouvent actuellement, du lundi au 


\section{Le glissement vers une énonciation ludique}

Le gommage de l'aspect politique des sujets et l'accent mis sur un spectacle plus "anodin" et sans engagement quant aux positions politiques, marque une tendance dans le paysage de l'information télévisuelle dans plusieurs émissions, et ceci depuis au moins une décennie. Un exemple caractéristique (quoique non systématique) se trouve dans l'émission politique intitulée Ellispontos, diffusée sur la chaîne privée Mega entre 1995 et 1996. Le dernier numéro de la saison est consacré aux noces du fils de l'ancien roi de Grèce à Londres et au débat vif et polémique, suscité par la décision de certains députés grecs d'y assister. À la fin de l'émission, la présentatrice Elli Stai, sur un ton manifestement ludique, porte un chapeau orné d'une voilette, tel que l'on aurait pu le porter au mariage en question, en nous souhaitant un bel été.

Toutefois, l'intégration du politique dans le domaine large du divertissement télévisuel se manifeste ces toutes dernières années par le biais de la participation de la classe politique au sein des émissions de divertissement. Cette tendance concerne un format d'émission spécifique que l'on pourrait qualifier d' "émission de divertissement musical du samedi soir". Il s'agit particulièrement de deux émissions diffusées le samedi soir, classées sous l'étiquette de divertissement ou de "show" par les chaînes et la presse télévisuelle. Leur concept pourrait se résumer en celui des émissions de plateau où le studio prend la forme d'un lieu public de réunion pour discuter de façon décontractée et écouter de la musique, telle une taverne avec de la musique en direct. La première s'intitule À la vôtre (en grec : Stin ygeia sas), diffusée sur la chaîne publique NET, présentée par l'acteur populaire Spyros Papadopoulos. La deuxième, s'intitulant Regarde ce que t'as fais (en grec: Koita ti ekanes), est diffusée sur la chaîne privée Alpha et présentée par la journaliste connue Semina Digeni. Les deux émissions en question sont considérées comme respectables et se positionnent aux antipodes des spectacles de divertissement qualifiés de "trash". Toutes les deux ont été primées, par ailleurs, lors des prix télévisuels grecs. C'est la raison pour laquelle, selon la presse télévisuelle, on a classé l'une (Regarde ce

vendredi, sur presque toutes les chaines de diffusion nationale. À titre d'exemple nous citons quelques unes : « Première ligne », diffusée à 5 h45 sur la chaîne publique NET ; « Bonjour avec Antenna » et « Bonjour la Grèce », diffusées respectivement à 5 h40 et 6 h30 sur la chaîne privée Antenna ; « Mega matinal », diffusée sur la chaîne privée Mega à 5 h45 ; « Nouveau jour », diffusée sur la chaîne privée Alpha à 5h30, etc. 
que t'as fais) dans la catégorie "émission de divertissement" et l'autre (À la vôtre) dans la catégorie "émission musicale", afin que toutes les deux puissent recevoir un prix.

Comme on pourrait l'imaginer, le discours politique, du moins dans sa forme la plus habituelle, ne trouve là aucune place. Le temps de parole, d'ailleurs, des invités en provenance de tous les domaines de la sphère publique, se trouve considérablement limité entre les chansons et les transitions des présentateurs qui tiennent le rôle d'hôte. Dans ce dispositif, simulant et revisitant des formes de divertissement externes à la télévision (par exemple la taverne musicale), l'on trouve de fortes consonances d'un mode de divertissement propre à la culture nationale grecque. En fait, ces programmes remplacent la sortie du samedi soir et aspirent à incarner une sorte de réponse "nationale" à des formes télévisuelles venues de l'étranger. Ils sont en quelque sorte, la réponse "à la grecque" aux reality shows de tous ordres (également populaires pour des tranches d'âges différentes) qui promeuvent une musique à succès considérée comme populiste et de moindre qualité.

Ces émissions se présentent implicitement comme les garants de certaines valeurs "nationales" ayant trait à la culture, à la socialisation, ainsi qu'à la tradition musicale grecques. Les personnalités politiques sont là pour cautionner le modèle de vie sociale représenté et offert par ce type de télévision. Certes, l'expression directe sur des sujets politiques n'a pas sa place dans cet univers; pour la première fois, peut-être, ce sont les hommes politiques qui remplissent, en quelque sorte, le rôle de "toile de fond" des émissions. Néanmoins, dans ces émissions qui affichent l'intention de préserver une tradition culturelle nationale, le personnel politique, par sa présence quasi-muette et par sa participation, fait acte de prescription du comportement social qui serait respectable, acceptable et montrable publiquement.

\section{En guise de conclusion}

L'ensemble des tendances observées dans les programmes télévisuels de ces dernières années à la télévision grecque n'est pas indépendant d'une évolution parallèle de la communication et du marketing politique qui ne cessent de se développer dans le pays. En ce sens, les pratiques grecques ne diffèrent pas énormément et sur le fond de celles rencontrées sur une échelle internationale. La mise en scène des personnalités politiques dans des activités qui dépassent le domaine politique (par exemple, la focalisation médiatique sur l'épouse 
du Premier ministre actuel Costas Karamanlis, notamment lors de la naissance de leurs enfants jumeaux) ou la création d'images - symboles (par exemple, la fameuse danse de zeïbekiko du président actuel du parti socialiste Georges Papandreou, ministre des affaires étrangères à l'époque, lors d'une rencontre avec son homologue turc entre l'île grecque de Samos et Kushadasi en Turquie, en juin 2001) témoigne d'une certaine continuité du phénomène qui ne traduit pas une pratique entièrement nouvelle. À ce sujet, une série d'images symboliques trouvent leur place dans la mémoire collective grecque. L'on se rappelle, par exemple, du traitement médiatique de la vie privée sentimentale d'Andreas Papandreou et de ses danses de zeïbekiko à lui, entre autres.

Certains entrevoient dans cette évolution la difficulté de distinguer les approches politiques des non politiques concernant les questions politiques et sociales (Pleios, 2002 : 279). Dans le même registre, Jean-Pierre Esquenazi affirme que «le politique ne sort pas indemne de ses rencontres avec la télévision » (Esquenazi, 1999 : 360), expliquant que cette dernière a participé aux transformations de la politique. D'autres, comme Jean Mouchon, proposent d'envisager la communication politique « autrement que comme technique opérationnelle dont on mesure l'efficacité au cas par cas » et de la considérer « comme une logique d'action influant sur la redéfinition de l'échange politique » (Mouchon, 1998 : 9). En ce qui concerne la Grèce, l'avènement de la télévision privée en 1990, qui s'inscrit dans un contexte socio-politique et idéologique précis, a, certes, modifié le paysage politico-médiatique. Toutefois, il ne s'agit pas d'inventer ex nihilio une nouvelle conception de la politique mais d'adapter le discours politique aux modes d'expression télévisuelle, processus qui, à son tour, entraîne des mutations structurelles plus ou moins profondes. 
